\title{
Rapid cardiac ventricular pacing to facilitate embolization of vein of Galen malformations: technical note
}

\author{
Aaron Hockley, MD, ${ }^{1}$ Michael K. Tso, MD, PhD, ${ }^{1}$ Mohammed A. Almekhlafi, MD, MSc, ${ }^{2}$ \\ Abhay K. Lodha, MBBS, MD, DM, MSc, ${ }^{3}$ Robin Clegg, MD, ${ }^{4}$ Jeremy Luntley, MBBS, ${ }^{5}$ \\ Muneer Eesa, MBBS, MD, ${ }^{6}$ and John H. Wong, MD, MSc ${ }^{1,6}$
}

\begin{abstract}
'Division of Neurosurgery, Department of Clinical Neurosciences, University of Calgary; ${ }^{2}$ Division of Neurology, Department of Clinical Neurosciences, University of Calgary; ${ }^{3}$ Division of Neonatology, Department of Paediatrics, University of Calgary; ${ }^{4}$ Division of Pediatric Cardiology, Department of Pediatrics and Libin Cardiovascular Institute of Alberta, University of Calgary; ${ }^{5}$ Division of Pediatric Anesthesia, Department of Anesthesia, and Division of Critical Care, Department of Pediatrics, University of Calgary; and ${ }^{6}$ Department of Radiology, University of Calgary, Alberta, Canada
\end{abstract}

\begin{abstract}
OBJECTIVE Vein of Galen aneurysmal malformations (VGAMs) in infancy have a poor natural history if left untreated. Their high-flow nature can preclude safe and accurate therapeutic vessel occlusion and the risk of inadvertent pulmonary embolism is predominant. The authors describe the technique of rapid cardiac ventricular pacing for inducing transient hypotension to facilitate the controlled embolization of VGAMs.
\end{abstract}

METHODS Initial transjugular venous access was obtained for placing temporary pacing leads for rapid cardiac ventricular pacing immediately prior to embolization. Definitive transarterial embolization procedures for the VGAMs were then performed in the same setting in which liquid embolic agents or coils were used.

RESULTS Beginning in 2010, a total of five procedures were performed in three infants. Transvenous rapid cardiac ventricular pacing was successfully achieved to induce systemic transient flow arrest in all but two attempts, and facilitated partial embolization with n-butyl cyanoacrylate (n-BCA) and coils in all procedures. Ventricular fibrillation occurred twice in one patient and was successfully reversed with defibrillation on both occasions. One patient failed to improve and died from refractory heart failure. Two patients stabilized following staged embolization.

CONCLUSIONS Rapid transvenous cardiac ventricular pacing can be considered to induce transient hypotension and facilitate controlled embolization in challenging high-flow VGAMs.

https://thejns.org/doi/abs/10.3171/2018.7.PEDS1852

KEYWORDS vein of Galen aneurysmal malformation; vascular malformation; embolization; ventricular pacing; surgical technique; vascular disorders

$\mathrm{C}$ EREBRAL arteriovenous malformations (AVMs) are a rare cause of neonatal heart failure. Vein of Galen aneurysmal malformations (VGAMs) represent $37 \%$ of all pediatric cerebral vascular malformations. ${ }^{1}$ These lesions can present with profound high-output cardiac failure due to the high-flow and low-pressure shunt, which creates a preferential pathway for a significant proportion (up to $80 \%$ ) of the cardiac output. Endovascular occlusion of the feeding arteries using liquid embolic agents such as n-butyl cyanoacrylate (n-BCA) has become the treatment of choice in appropriately selected patients. ${ }^{6}$
Given the high-flow nature of these shunts, endovascular embolization can be technically challenging with significant risk of distal migration of the embolic agent with resultant inadvertent venous outflow occlusion and/or pulmonary embolism with disastrous consequences.

We describe our technique using transvenous rapid cardiac ventricular pacing to induce transient controlled global hypotension with reduced pulse pressure, and facilitate appropriate delivery of embolic agents in a more controlled manner. This technique has been previously described for optimizing coronary and thoracic stent placement, cardiac

ABBREVIATIONS AVM = arteriovenous malformation; MAP = mean arterial pressure; $n-B C A=n$-butyl cyanoacrylate; $N I C U=$ neonatal intensive care unit; $R V O T=$ right ventricle outflow tract; VGAM = vein of Galen aneurysmal malformation.

SUBMITTED January 24, 2018. ACCEPTED July 10, 2018.

INCLUDE WHEN CITING Published online October 5, 2018; DOI: 10.3171/2018.7.PEDS1852. 
valve placement, and neurosurgical procedures such as microsurgical clipping of intracranial aneurysms, resection of brain AVMs, and a case of VGAM.2,4,8-12

\section{Methods}

This study was a retrospective review of a prospectively maintained database of pediatric vascular lesions treated by endovascular therapy. A total of 5 procedures in 3 patients were identified in which rapid cardiac ventricular pacing was used for assistance with the embolization procedure. Our first case was performed in November 2010.

\section{Endovascular Procedure}

All procedures were performed with the patient under general anesthesia supported by the pediatric anesthesiology team with experience in managing pediatric patients with cerebral vascular malformations. A 4-Fr femoral arterial sheath was used in all cases for the embolization component of the procedures. A 4-Fr Terumo Glidecath catheter (Terumo Medical Corporation) was used as a guiding catheter. A detachable tip microcatheter (Apollo, ev3 Neurovascular) was used for embolization with liquid embolic agents while an Echelon-10 microcatheter (ev3 Neurovascular) was used for coil embolization. Concentrated $(60 \%-80 \%)$ n-BCA was used for embolization in four procedures while a combination of Cashmere, Micrusphere, and Deltapaq detachable microcoils (Codman Neuro) were used in one procedure.

\section{Pacing Technique}

A right ventricular transvenous pacemaker for rapid right ventricular pacing was inserted under fluoroscopic guidance by interventional cardiology (Fig. 1). Pacing was induced via an external pacemaker connected to the implanted electrode near the right ventricle outflow tract (RVOT). For the first case, a passive (nonfixed) 5-Fr pacing device and a Swan-Ganz bipolar pacing catheter (Edwards Lifesciences) were used. Due to the risk of unpredictable capture, a $200-\mathrm{cm}$ fixed 5-Fr screw-in model 6416 Temporary Transvenous Pacing Lead System (Medtronic) was used for subsequent procedures. The devices were inserted through the right internal jugular vein via a 6-Fr sheath introducer (Cordis Corporation). Additionally, defibrillator electrodes were placed and connected preemptively prior to patient positioning on the angiography table. Once the pacing capture threshold was established, a double-the-threshold voltage was used for pacing voltage discharge. A heart rate that reduced the mean arterial pressure (MAP) to $<30 \mathrm{~mm} \mathrm{Hg}$ with a pulseless arterial line tracing (correlating with 220-240 beats per minute) was targeted (Fig. 2). Rapid ventricular pacing was maintained for a maximum of 30-45 seconds at the discretion of the cardiologist or until an abnormal rhythm occurred. Prior to injecting n-BCA, pacing wire capture testing was performed in all cases to ensure pacing functionality, sustained myocardial capture, MAP response, and appropriate communication between team members. The induced ventricular tachycardia was considered successful if it resulted in momentary hypotension with $\mathrm{MAP}<30 \mathrm{~mm} \mathrm{Hg}$.

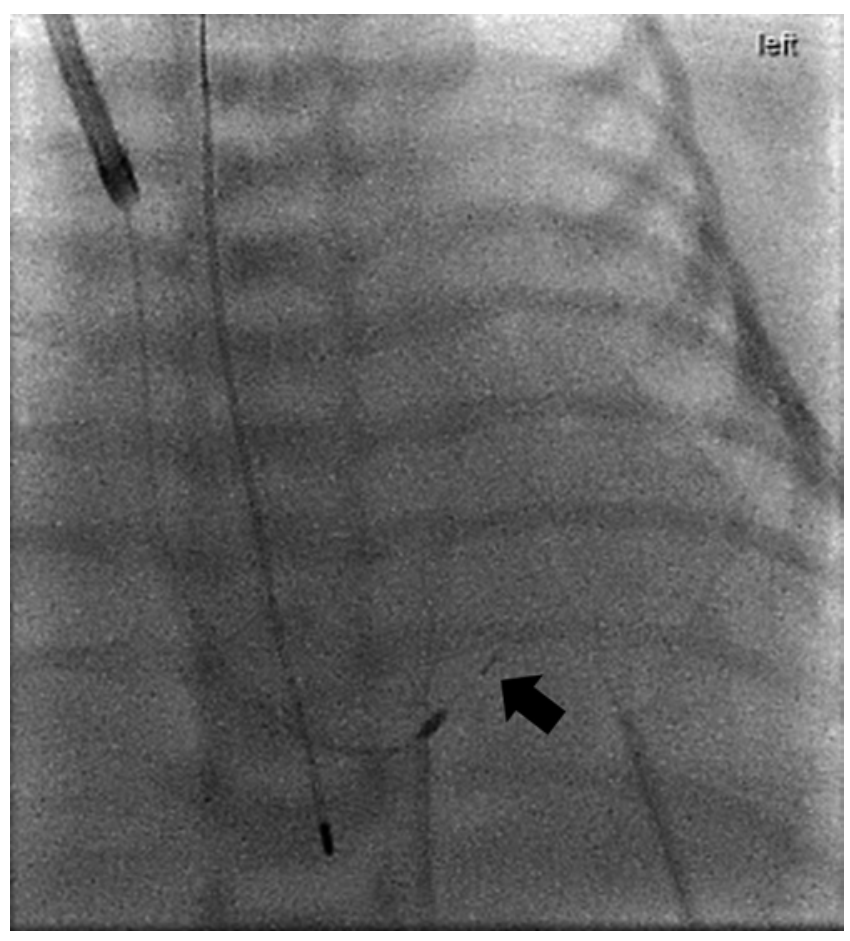

FIG. 1. Frontal spot film obtained during fluoroscopy demonstrating the tip of the fixed pacing wire (arrow) at the expected location of the RVOT.

\section{Illustrative Cases Case 1}

A full-term male neonate was admitted to the neonatal intensive care unit (NICU) on day 2 of life, presenting with a heart murmur and tachypnea. An echocardiogram revealed a very large aortic arch with retrograde flow in the aorta. Imaging with ultrasonography followed by MRI confirmed a mural-type VGAM (Lasjaunias classification) or type 3 VGAM (Yaşargil classification). ${ }^{5,13}$ The patient developed high-output cardiac failure requiring intubation and inotropic support. He also had moderate renal dysfunction and mild coagulopathy, and was receiving intravenous antibiotics for sepsis. Despite the poor clinical picture, after discussions among the multidisciplinary team members and family, a decision was made to proceed with treatment. Treatment of his VGAM was undertaken at the age of 8 days by embolization of two large arterial pedicles from the posterior circulation. Rapid ventricular pacing was successfully achieved in one of the two attempts. The failure of the second attempt was likely due to the passive nature of the pacing wire, which lost myocardial contact, resulting in only intermittent pacing capture. A moderate decrease in flow through the fistula was achieved after embolization. Final control images showed an unexpected, small, nonocclusive n-BCA cast in the distal basilar artery likely due to inadvertent embolization. Urgent MRI showed acute infarcts in both thalami and extensive intraventricular and subarachnoid hemorrhage with associated obstructive hydrocephalus. The patient developed multiorgan failure along with progressive increase in the size of the VGAM. An extremely 

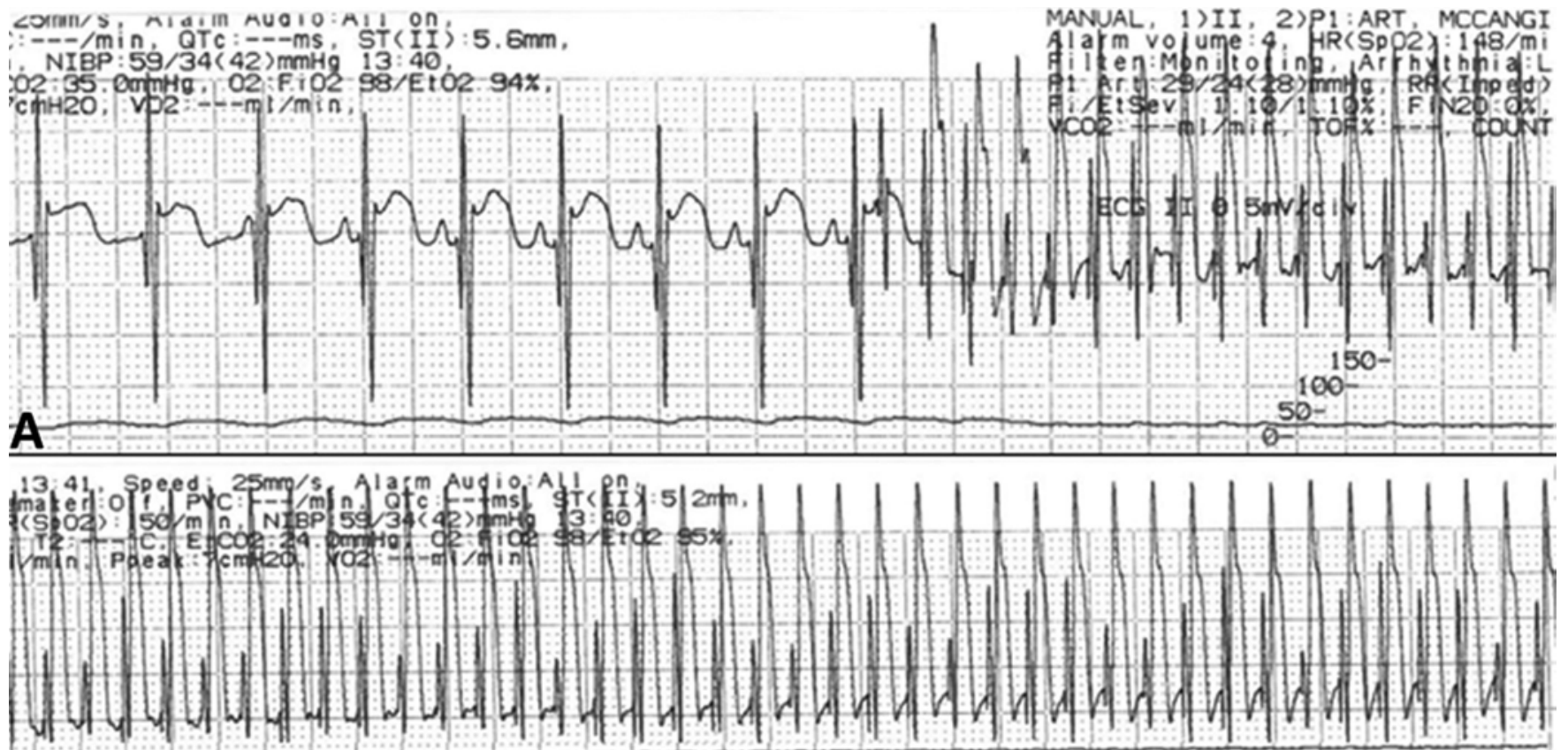

\section{B}

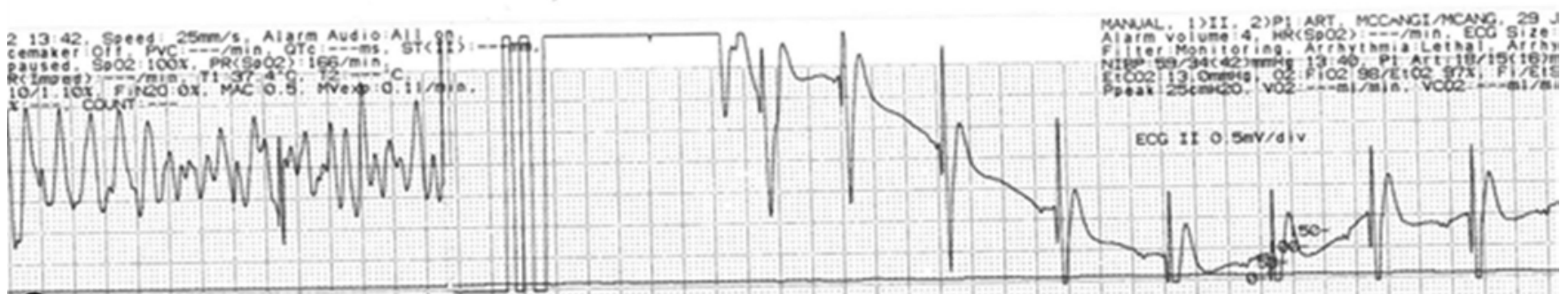

C

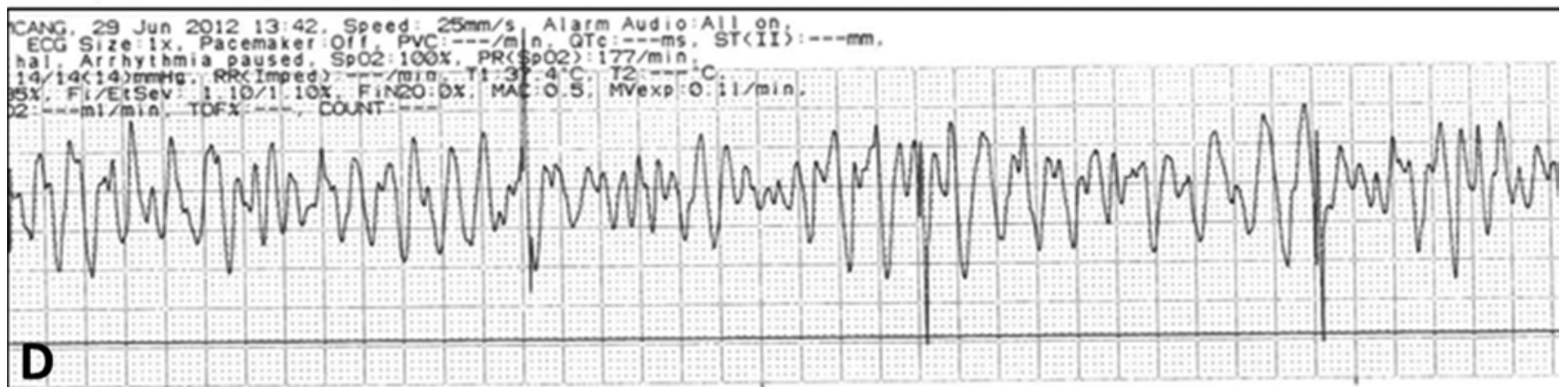

FIG. 2. Electrocardiography tracings demonstrating baseline tracing with onset of rapid pacing $(\mathbf{A})$ and sustained capture during embolization (B) followed by return to spontaneous rhythm following cessation of pacing (C). In case 2 ventricular fibrillation occurred following cessation of rapid pacing (D).

poor prognosis led to withdrawal of life-sustaining therapy and he died at the age of 2 weeks.

\section{Case 2}

A 10-day-old male full-term neonate was noted to have a loud cranial bruit, audible without a stethoscope, immediately after birth. He rapidly developed severe heart failure and was admitted to the NICU. Imaging revealed a high-flow arteriovenous fistula (type IV VGAM, Yaşargil classification) with multiple hypertrophied arterial feeders draining into a dilated venous pouch deep to the hippocampal formation, which finally drained into a markedly dilated vein of Galen. The decision was made to proceed with endovascular embolization.

This first procedure was performed at the age of 10 days with embolization via the right posterior cerebral 

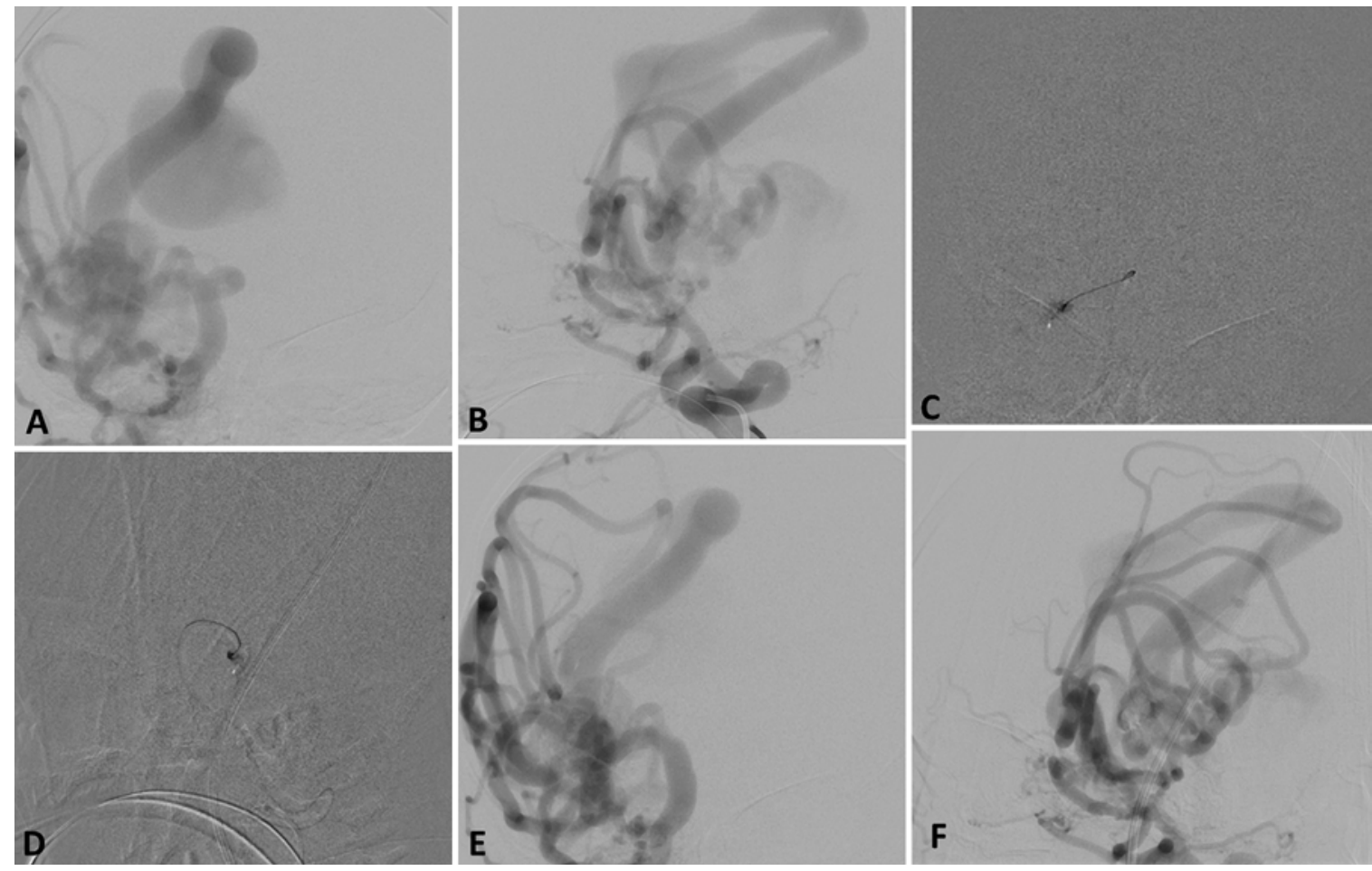

FIG. 3. Case 2. First embolization procedure in 10-day-old neonate with type IV Yaşargil VGAM. Anteroposterior (A) and lateral (B) projections of a right internal carotid artery injection showed a high-flow arteriovenous fistula ultimately draining into a dilated vein of Galen. After institution of rapid cardiac ventricular pacing, n-BCA material was injected into the distal right anterior choroidal artery feeding the fistula, in a controlled manner, as seen in the anteroposterior (C) and lateral projections (D). After 2 additional embolizations of arterial feeders, final right internal carotid artery injection anteroposterior $(\mathbf{E})$ and lateral $(\mathbf{F})$ projections showed decreased flow into the fistula.

and anterior choroidal arteries (Fig. 3). In this case, a 6-Fr introducer sheath (Cordis Corporation) was placed in the right internal jugular vein, and a 200-cm fixed 5-Fr screwin model 6416 Temporary Transvenous Pacing Lead System was fixed into the RVOT. Seven trial episodes of right ventricle overdrive pacing were performed. After the first three failed attempts during pacing wire capture testing, the pacing wire was repositioned under fluoroscopy. This resulted in consistent capture during the subsequent four attempts. Two of those trials resulted in ventricular fibrillation that responded to defibrillation. A final angiographic run showed significant reduction of the fistulous communication and venous drainage with associated significant clinical improvement in the patient's hemodynamic status postoperatively.

A second procedure was performed at the age of 8 months due to mild hydrocephalus and significant flow through the VGAM. Pacing was successful in two of three attempts. During the second embolization attempt, 2-to-1 capture (pacing twice as fast as the recorded heart rate) occurred despite pacing at the same rate and output, possibly due to a change in ventricular refractoriness associated with a prolonged anesthetic or metabolic disturbance. Decreasing the pacing rate by $10 \mathrm{bpm}$ overcame this problem. During the last embolization injection, the microcatheter could not be released as the n-BCA had already polymerized. The microcatheter was cut at its hub at the groin level under gentle tension and released into the iliac system. Final runs showed residual slow shunting through the VGAM. Postprocedure, the patient received anticoagulation therapy and maintained normal neurological development.

At the age of 22 months, a third embolization procedure using endovascular coil deployment in conjunction with rapid cardiac ventricular pacing was successful in further reducing the flow through the fistula. This procedure involved the coil embolization of three arterial feeders supplying the fistula. In this procedure all attempts at pacing were successful in MAP reduction and return of sinus rhythm. Final angiographic runs showed slow residual filling of the VGAM. MRI performed following the procedure demonstrated significant reduction of flow toward the fistula and patency of the dural venous sinuses.

\section{Case 3}

A 4-month-old male infant presented to the community pediatrician with increasing head circumference and mild failure to thrive. A cranial sonogram revealed features suspicious for a VGAM, which was confirmed with MRI. He was diagnosed with a high-flow mural-type VGAM (Lasjaunias classification). The patient was treated at 5 months of age with a transarterial endovascular approach. The lesion was supplied by a large posterior choroidal branch from the left PCA. Right internal jugular venous access 
was obtained for placing the temporary pacing leads as in our prior cases. After pacing wire capture testing, three attempts at overdrive pacing resulted in capture every time. Embolization was sequentially performed during those three attempts using high-concentration n-BCA. No episodes of ventricular fibrillation were noted.

\section{Discussion}

Embolization of pediatric cerebral vascular malformations requires precise placement of embolic material, which can be particularly difficult in high-flow lesions. Rapid cardiac ventricular pacing is a well-described adjunct in the setting of interventional cardiology and has also been sparingly described in the neurosurgical literature with respect to intracranial aneurysm and AVM microsurgery. ${ }^{9,11,12}$ Ramgren and colleagues have recently published a case report of using rapid ventricular pacing as an adjunct in the embolization of a high-flow VGAM in a neonate presenting with heart failure..$^{10}$ In this paper we demonstrate the evolution of the technique of rapid ventricular pacing in VGAM embolization over a small case series, highlighting some of the progress and pitfalls noted when adopting a new technique.

Rapid ventricular pacing induces a controlled ventricular tachycardia and the resultant rapid heart rate causes a significant decrease in ventricular filling time, causing reduced stroke volume and cardiac output. This has been shown to cause abrupt and profound hypotension, but it is also rapidly reversible, thus making it an attractive tool for the prevention of unwanted distal embolization in the setting of high-flow lesions. ${ }^{3}$ The overdrive pacing has a predictable self-limiting response with rapid return of sinus rhythm upon cessation of pacing. This technique contrasts with other previously described pharmacological techniques using intravenous agents such as esmolol or adenosine. Adenosine has a variable onset speed and duration of asystole and is prone to ectopic ventricular beats, whereas esmolol has an onset and offset on the order of minutes. ${ }^{12}$ In addition, these agents do not cause cardiac standstill, and can require additional medications to obtain the desired reduction in arterial pressures. ${ }^{7}$ Overdrive pacing provides transient but sustained hypotension on the order of seconds, therefore making it ideally suited for rapid endovascular injection of embolic agents.

Due to the minimally invasive nature of endovascular treatments, any additional maneuvers that can be used to control the delivery of devices can potentially add to the overall safety of the procedures and may improve clinical outcomes. Nienaber et al. used rapid right ventricular pacing in 27 patients in their series to assist with thoracic endograft placement. ${ }^{8}$ This technique hypothetically allowed the decrease in occurrence of the windsock effect during thoracic aortic stent-graft placement. In their adult population the authors describe the risk of ventricular tachyarrhythmia to be less than $1 \%{ }^{8}$ The risk of right atrial or ventricular tachyarrhythmia is also low if the procedure is performed under fluoroscopy. Billings and colleagues described the use of rapid ventricular pacing in their series of patients who underwent transcatheter aortic valve implantations. ${ }^{2}$ Rapid ventricular pacing in combination with temporary interruption of mechanical ventilation helped limit displacement of the balloon during inflation and implantation of the aortic valvular prosthesis.

As opposed to the adult population, the risks related to rapid cardiac ventricular pacing, including iatrogenic ventricular fibrillation, are likely substantially greater in pediatric patients, particularly medically and neurologically unstable neonates, and require the close attention of the pediatric anesthesiologist and cardiologist during the procedure. Given the high degree of complexity and risk of the overall procedure, efficient and accurate communication between the multidisciplinary team members is essential to maximize safety. This was constantly emphasized during the capture testing runs and prior to each injection. Recent technical adjuncts such as higher-viscosity embolic agents, compatible dual-lumen balloon-tipped microcatheters, and detachable microcatheters may offer alternative strategies for the controlled embolization of high-flow arteriovenous lesions. Whether such strategies can be utilized safely in the medically tenuous neonatal population affected by VGAMs remains to be fully elucidated. Our cases highlight the benefit of MAP reduction by induced hypotension from rapid pacing, namely accurate delivery and polymerization of the n-BCA within the target vessels. We have also inadvertently encountered some of the related pitfalls of using rapid pacing. During our first case we lost the ability to pace, and this was presumed to be secondary to the passive nature of the inserted pacing wire. These episodes were somewhat anticipated given that the patient (and therefore the myocardium) presented in extremis. Our second patient experienced two episodes of transient ventricular fibrillation secondary to the rapid ventricular pacing. There were no immediate or long-term procedural cardiac complications identified secondary to the use of the rapid ventricular pacing.

\section{Conclusions}

High-flow AVMs including VGAMs can pose technical challenges for optimal delivery of embolic materials. In this case series, we demonstrated the utility as well as pitfalls of rapid cardiac ventricular pacing for inducing hypotension to aid in controlled embolization. The cautious application of this method with a multidisciplinary team of experienced operators may be useful in challenging cases.

\section{References}

1. Berenstein A, Ortiz R, Niimi Y, Elijovich L, Fifi J, Madrid $\mathrm{M}$, et al: Endovascular management of arteriovenous malformations and other intracranial arteriovenous shunts in neonates, infants, and children. Childs Nerv Syst 26:1345-1358, 2010

2. Billings FT IV, Kodali SK, Shanewise JS: Transcatheter aortic valve implantation: anesthetic considerations. Anesth Analg 108: 1453-1462, 2009

3. Daehnert I, Rotzsch C, Wiener M, Schneider P: Rapid right ventricular pacing is an alternative to adenosine in catheter interventional procedures for congenital heart disease. Heart 90:1047-1050, 2004

4. Lasa G, Larman M, Gaviria K, Sanmartín JC, Sádaba M, Rumoroso JR: Coronary stent immobilization during angio- 
plasty by transcoronary ventricular pacing via a guidewire. Rev Esp Cardiol 62:288-292, 2009

5. Lasjaunias P, Rodesch G, Pruvost P, Laroche FG, Landrieu P: Treatment of vein of Galen aneurysmal malformation. J Neurosurg 70:746-750, 1989

6. Lasjaunias PL, Chng SM, Sachet M, Alvarez H, Rodesch G, Garcia-Monaco R: The management of vein of Galen aneurysmal malformations. Neurosurgery 59 (5 Suppl 3):S184S194, S3-S13, 2006

7. Mehta C, Desai T, Shebani S, Stickley J, DE Giovanni J: Rapid ventricular pacing for catheter interventions in congenital aortic stenosis and coarctation: effectiveness, safety, and rate titration for optimal results. J Interv Cardiol 23:7-13, 2010

8. Nienaber CA, Kische S, Rehders TC, Schneider H, Chatterjee $\mathrm{T}$, Bünger CM, et al: Rapid pacing for better placing: comparison of techniques for precise deployment of endografts in the thoracic aorta. J Endovasc Ther 14:506-512, 2007

9. Nimjee SM, Smith TP, Kanter RJ, Ames W, Machovec KA, Grant GA, et al: Rapid ventricular pacing for a basilar artery pseudoaneurysm in a pediatric patient: case report. J Neurosurg Pediatr 15:625-629, 2015

10. Ramgren B, Rask O, Gelberg J, Liuba P, Undrén P, Wassélius J: Endovascular treatment of vein of Galen aneurysmal malformation using rapid ventricular pacing: a case report. Interv Neuroradiol 23:97-101, 2017

11. Saldien V, Menovsky T, Rommens M, Van der Steen G, Van Loock K, Vermeersch G, et al: Rapid ventricular pacing for flow arrest during cerebrovascular surgery: revival of an old concept. Neurosurgery 70 (2 Suppl Operative):270-275, 2012

12. Whiteley JR, Payne R, Rodriguez-Diaz C, Ellegala DB, Reeves ST: Rapid ventricular pacing: a novel technique to decrease cardiac output for giant basilar aneurysm surgery. J Clin Anesth 24:656-658, 2012

13. Yasargil MG, Antic J, Laciga R, Jain KK, Boone SC: Arteriovenous malformations of vein of Galen: microsurgical treatment. Surg Neurol 3:195-200, 1976

\section{Disclosures}

The authors report no conflict of interest concerning the materials or methods used in this study or the findings specified in this paper.

\section{Author Contributions}

Conception and design: Wong, Eesa. Drafting the article: Wong, Hockley, Tso, Eesa. Critically revising the article: Wong, Hockley, Tso, Almekhalfi, Lodha, Clegg, Eesa. Reviewed submitted version of manuscript: all authors. Approved the final version of the manuscript on behalf of all authors: Wong. Statistical analysis: Luntley. Study supervision: Wong.

\section{Supplemental Information}

Current Affiliations

Dr. Aaron Hockley: Division of Neurosurgery, Department of Surgery, University of Alberta, AB, Canada.

\section{Correspondence}

John H. Wong: Foothills Medical Centre, Calgary, AB, Canada. jwong@ucalgary.ca. 